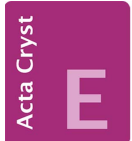
COMMUNICATIONS

ISSN 2056-9890

\section{Crystal structure of metobromuron}

\author{
Gihaeng Kang, Jineun Kim, ${ }^{*}$ Eunjin Kwon and Tae Ho \\ Kim*
}

Department of Chemistry and Research Institute of Natural Sciences, Gyeongsang National University, Jinju 660-701, Republic of Korea. *Correspondence e-mail:

thkim@gnu.ac.kr, jekim@gnu.ac.kr

Received 6 July 2015; accepted 14 July 2015

Edited by P. C. Healy, Griffith University, Australia

The title compound [systematic name: 3-(4-bromophenyl)-1methoxy-1-methylurea], $\mathrm{C}_{9} \mathrm{H}_{11} \mathrm{BrN}_{2} \mathrm{O}_{2}$, is a phenylurea herbicide. The dihedral angle between the plane of the urea group and that of the bromophenyl ring is $39.13(10)^{\circ}$. In the crystal, $\mathrm{N}-\mathrm{H} \cdots \mathrm{O}$ and $\mathrm{C}-\mathrm{H} \cdots \mathrm{O}$ hydrogen bonds and weak $\mathrm{C}-$ $\mathrm{H} \cdots \pi$ interactions link adjacent molecules, forming chains along the $a$-axis direction. In addition, short intermolecular $\mathrm{Br} \cdots \mathrm{Br}$ contacts [3.648 (4) $\AA$ ] are present, resulting in a twodimensional network extending parallel to (101).

Keywords: crystal structure; metobromuron; phenylurea herbicide; hydrogen bonding; $\mathrm{Br} \cdots \mathrm{Br}$ contacts.

CCDC reference: 1412609

\section{Related literature}

For information on the herbicidal properties of the title compound, see: Leila et al. (2011). For related crystal structures, see: Black et al. (2010); Kostyanovsky et al. (2010).

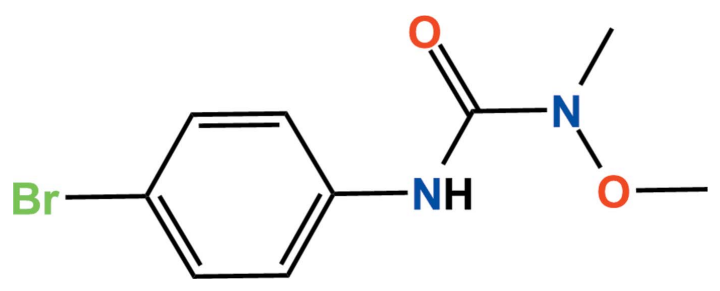

\section{Experimental}

\subsection{Crystal data}

\section{$\mathrm{C}_{9} \mathrm{H}_{11} \mathrm{BrN}_{2} \mathrm{O}_{2}$}

$M_{r}=259.11$
Orthorhombic, $\mathrm{Pbca}$ $a=9.8184$ (2) $\AA$ $b=11.3286(3) \AA$

$c=18.9569(5) \AA$

$V=2108.55(9) \AA^{3}$

$Z=8$

2.2. Data collection

Bruker APEXII CCD diffractometer

Absorption correction: multi-scan (SADABS; Bruker, 2013)

$T_{\min }=0.389, T_{\max }=0.927$

\subsection{Refinement}

$R\left[F^{2}>2 \sigma\left(F^{2}\right)\right]=0.029$

$w R\left(F^{2}\right)=0.066$

$S=1.03$

2424 reflections

129 parameters

$\mathrm{H}$-atom parameters constrained

$\Delta \rho_{\max }=0.41{\mathrm{e} \AA^{-3}}^{-3}$

$\Delta \rho_{\min }=-0.42 \mathrm{e}^{-3}$
Table 1

Hydrogen-bond geometry $\left(\AA,^{\circ}\right)$.

$C g 1$ is the centroid of the C1-C6 ring.

\begin{tabular}{lllll}
\hline$D-\mathrm{H} \cdots A$ & $D-\mathrm{H}$ & $\mathrm{H} \cdots A$ & $D \cdots A$ & $D-\mathrm{H} \cdots A$ \\
\hline $\mathrm{N} 1-\mathrm{H} 1 N \cdots \mathrm{O} 1^{\mathrm{i}}$ & 0.88 & 2.39 & $3.130(2)$ & 142 \\
$\mathrm{C} 2-\mathrm{H} 2 \cdots \mathrm{O} 1^{\mathrm{i}}$ & 0.95 & 2.42 & $3.217(3)$ & 142 \\
$\mathrm{C} 9-\mathrm{H} 9 A \cdots C g 1^{\mathrm{i}}$ & 0.98 & 2.99 & $3.477(3)$ & 112 \\
\hline
\end{tabular}

Symmetry code: (i) $x-\frac{1}{2}, y,-z+\frac{3}{2}$.

Data collection: APEX2 (Bruker, 2013); cell refinement: SAINT (Bruker, 2013); data reduction: $S A I N T$; $\operatorname{program}(\mathrm{s})$ used to solve structure: SHELXS97 (Sheldrick 2008); program(s) used to refine structure: SHELXL2013 (Sheldrick, 2015); molecular graphics: DIAMOND (Brandenburg, 2010); software used to prepare material for publication: SHELXTL (Sheldrick, 2008).

\section{Acknowledgements}

This research was supported by the Basic Science Research Program through the National Research Foundation of Korea (NRF) funded by the Ministry of Education, Science and Technology (No. 2014R1A1A4A01009105).

Supporting information for this paper is available from the IUCr electronic archives (Reference: HG5451).

\section{References}

Black, H. M. \& Baughman, R. G. (2010). Acta Cryst. E66, o2221.

Brandenburg, K. (2010). DIAMOND. Crystal Impact GbR, Bonn, Germany. Bruker (2013). APEX2, SAINT and SADABS. Bruker AXS Inc., Madison, Wisconsin, USA.

Kostyanovsky, R. G., Shtamburg, V. G., Shishkin, O. V., Zubatyuk, R. I., Shtamburg, V. V., Anishchenko, A. A. \& Mazepa, A. V. (2010). Mendeleev Commun. 20, 167-169.

Leila, N., Sakina, H., Abdelaziz, B., Fatiha, M. \& Fateh, L. L. D. (2011). J. Biol. Sci. 11, 1-9.

Sheldrick, G. M. (2008). Acta Cryst. A64, 112-122.

Sheldrick, G. M. (2015). Acta Cryst. C71, 3-8. 


\section{supporting information}

Acta Cryst. (2015). E71, o589 [https://doi.org/10.1107/S205698901501347X]

\section{Crystal structure of metobromuron}

\section{Gihaeng Kang, Jineun Kim, Eunjin Kwon and Tae Ho Kim}

\section{S1. Comment}

Metobromuron: 3-(4-bromophenyl)-1-methoxy-1-methylurea, is a phenylurea herbicide and has been used for the control of broadleaf weeds in cereal and vegetable crops, acting through the inhibition of photosynthesis (Leila et al., 2011).

However, until now its crystal structure has not been reported. In the title compound (Fig. 1), the dihedral angle between the plane of the urea group and the bromophenyl ring is $39.13(10)^{\circ}$. All bond lengths and bond angles are normal and comparable to those observed in similar crystal structures (Black et al., 2010; Kostyanovsky et al., 2010).

In the crystal structure (Fig. 2), $\mathrm{N}-\mathrm{H} \cdots \mathrm{O}$ and $\mathrm{C}-\mathrm{H} \cdots \mathrm{O}$ hydrogen bonds and weak $\mathrm{C}-\mathrm{H} \cdots \pi$ interactions link adjacent molecules (Table 1), forming one-dimensional chains along the $a$-axis direction. In addition, weak intermolecular short $\mathrm{Br} \cdots \mathrm{Br}$ contacts $[3.648$ (4) $\AA]$ are present, resulting in a two-dimensional network extending parallel to (101).

\section{S2. Experimental}

The title compound was purchased from the Dr. Ehrenstorfer GmbH Company. Slow evaporation of a solution in ethyl acetate gave single crystals suitable for X-ray analysis.

\section{S3. Refinement}

All H-atoms were positioned geometrically and refined using a riding model with $\mathrm{d}(\mathrm{N}-\mathrm{H})=0.88 \AA, U_{\text {iso }}=1.2 U_{\text {eq }}(\mathrm{C})$ for urea $\mathrm{N}-\mathrm{H}, \mathrm{d}(\mathrm{C}-\mathrm{H})=0.98 \AA, U_{\text {iso }}=1.5 U_{\text {eq }}(\mathrm{C})$ for methyl group, $\mathrm{d}(\mathrm{C}-\mathrm{H})=0.95 \AA, U_{\text {iso }}=1.2 U_{\text {eq }}(\mathrm{C})$ for aromatic $\mathrm{C}-$ H.

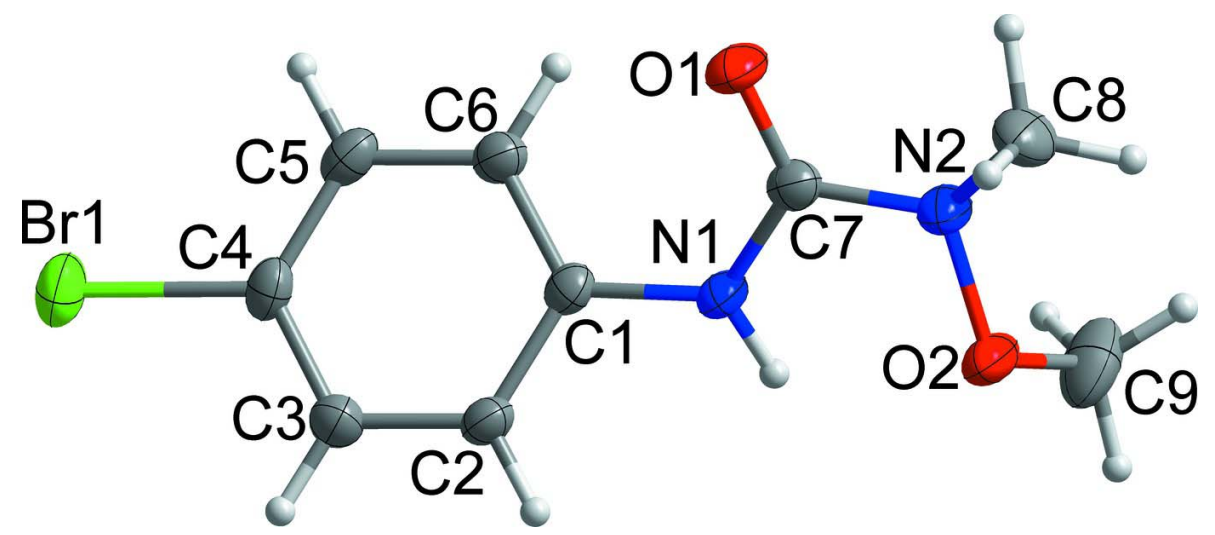

Figure 1

The asymmetric unit of the title compound with the atom numbering scheme. Displacement ellipsoids are drawn at the $50 \%$ probability level. $\mathrm{H}$ atoms are shown as small spheres of arbitrary radius. 


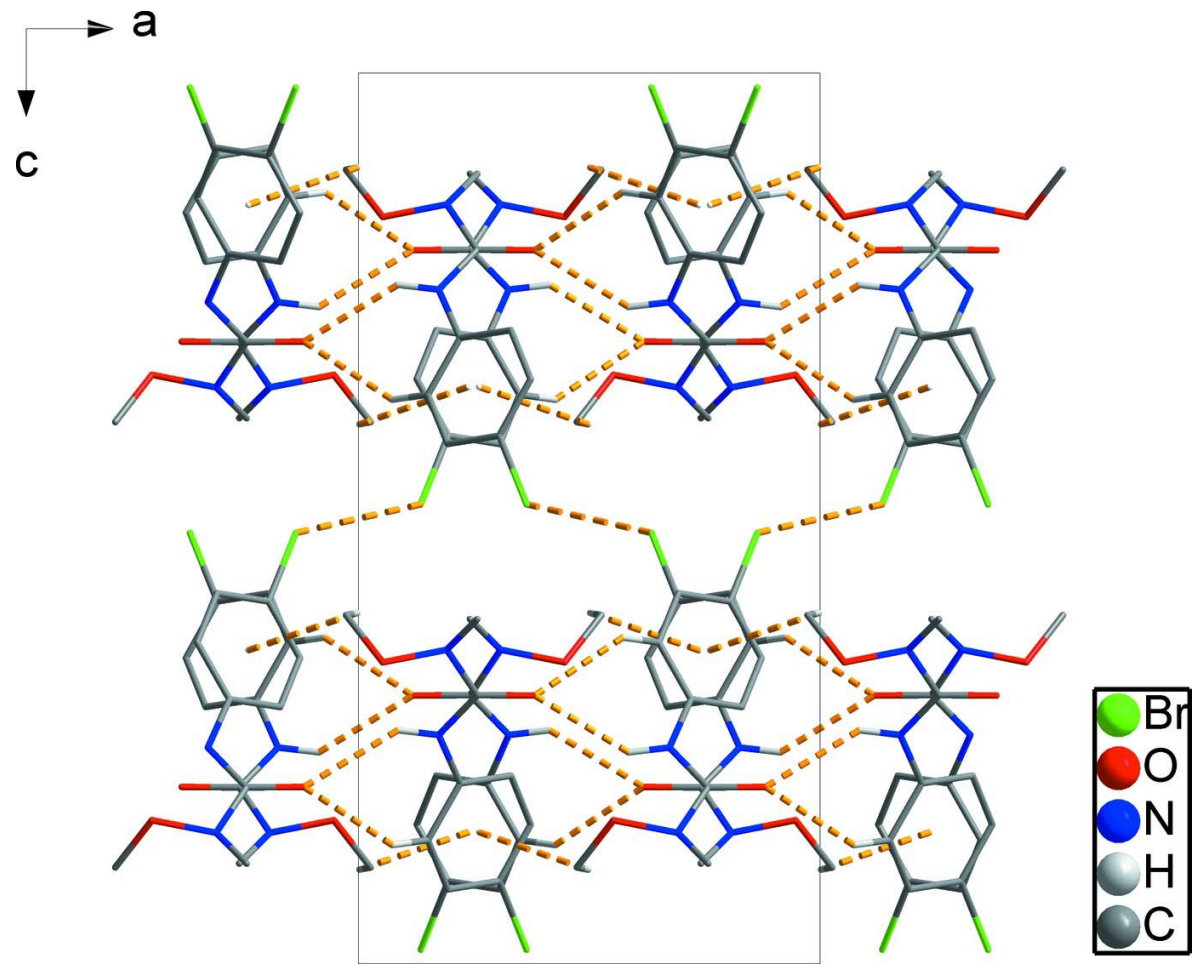

Figure 2

Crystal packing viewed along the $b$ axis. The intermolecular interactions are shown as dashed lines.

\section{3-(4-Bromophenyl)-1-methoxy-1-methylurea}

\section{Crystal data}

$\mathrm{C}_{9} \mathrm{H}_{11} \mathrm{BrN}_{2} \mathrm{O}_{2}$

$M_{r}=259.11$

Orthorhombic, $\mathrm{Pbca}$

$a=9.8184(2) \AA$

$b=11.3286(3) \AA$

$c=18.9569(5) \AA$

$V=2108.55(9) \AA^{3}$

$Z=8$

$F(000)=1040$

\section{Data collection}

Bruker APEXII CCD diffractometer

$\varphi$ and $\omega$ scans

Absorption correction: multi-scan

(SADABS; Bruker, 2013)

$T_{\min }=0.389, T_{\max }=0.927$

17922 measured reflections

\section{Refinement}

Refinement on $F^{2}$

Least-squares matrix: full

$R\left[F^{2}>2 \sigma\left(F^{2}\right)\right]=0.029$

$w R\left(F^{2}\right)=0.066$
$D_{\mathrm{x}}=1.632 \mathrm{Mg} \mathrm{m}^{-3}$

Mo $K \alpha$ radiation, $\lambda=0.71073 \AA$

Cell parameters from 3544 reflections

$\theta=3.0-24.1^{\circ}$

$\mu=3.88 \mathrm{~mm}^{-1}$

$T=173 \mathrm{~K}$

Plate, colourless

$0.30 \times 0.16 \times 0.02 \mathrm{~mm}$

2424 independent reflections

1857 reflections with $I>2 \sigma(I)$

$R_{\text {int }}=0.048$

$\theta_{\text {max }}=27.5^{\circ}, \theta_{\min }=2.2^{\circ}$

$h=-12 \rightarrow 12$

$k=-14 \rightarrow 14$

$l=-23 \rightarrow 24$

$S=1.03$

2424 reflections

129 parameters

0 restraints 
Hydrogen site location: inferred from neighbouring sites

H-atom parameters constrained

$$
\begin{aligned}
& w=1 /\left[\sigma^{2}\left(F_{\mathrm{o}}^{2}\right)+(0.0255 P)^{2}+1.105 P\right] \\
& \text { where } P=\left(F_{\mathrm{o}}^{2}+2 F_{\mathrm{c}}{ }^{2}\right) / 3 \\
& (\Delta / \sigma)_{\max }=0.001 \\
& \Delta \rho_{\max }=0.41 \mathrm{e} \AA^{-3} \\
& \Delta \rho_{\min }=-0.42 \mathrm{e} \AA^{-3}
\end{aligned}
$$

Special details

Geometry. All e.s.d.'s (except the e.s.d. in the dihedral angle between two 1.s. planes) are estimated using the full

\begin{tabular}{|c|c|c|c|c|}
\hline & $x$ & $y$ & $z$ & $U_{\text {iso }} * / U_{\text {eq }}$ \\
\hline Br1 & $0.86464(3)$ & $0.10694(2)$ & $0.51608(2)$ & $0.03673(10)$ \\
\hline $\mathrm{O} 1$ & $0.88255(14)$ & $0.47320(14)$ & $0.80131(9)$ & $0.0337(4)$ \\
\hline $\mathrm{O} 2$ & $0.55297(15)$ & $0.56454(15)$ & $0.83862(9)$ & $0.0338(4)$ \\
\hline N1 & $0.67399(18)$ & $0.41832(16)$ & $0.75709(10)$ & $0.0259(4)$ \\
\hline $\mathrm{H} 1 \mathrm{~N}$ & 0.5855 & 0.4269 & 0.7624 & $0.031 *$ \\
\hline N2 & $0.69171(18)$ & $0.54270(17)$ & $0.85274(10)$ & $0.0297(5)$ \\
\hline $\mathrm{C} 1$ & $0.7206(2)$ & $0.34339(18)$ & $0.70276(12)$ & $0.0224(5)$ \\
\hline $\mathrm{C} 2$ & $0.6508(2)$ & 0.34425 (19) & $0.63907(12)$ & $0.0249(5)$ \\
\hline $\mathrm{H} 2$ & 0.5732 & 0.3934 & 0.6335 & $0.030 *$ \\
\hline $\mathrm{C} 3$ & $0.6936(2)$ & $0.2741(2)$ & $0.58381(12)$ & $0.0276(5)$ \\
\hline H3 & 0.6467 & 0.2756 & 0.5400 & $0.033 *$ \\
\hline $\mathrm{C} 4$ & $0.8053(2)$ & 0.20197 (19) & $0.59297(12)$ & $0.0265(5)$ \\
\hline $\mathrm{C} 5$ & $0.8734(2)$ & 0.19632 (19) & $0.65655(12)$ & $0.0278(5)$ \\
\hline H5 & 0.9485 & 0.1444 & 0.6625 & $0.033 *$ \\
\hline C6 & $0.8306(2)$ & $0.26736(19)$ & $0.71147(12)$ & $0.0264(5)$ \\
\hline H6 & 0.8766 & 0.2643 & 0.7555 & $0.032 *$ \\
\hline $\mathrm{C} 7$ & $0.7577(2)$ & 0.47817 (19) & $0.80181(12)$ & $0.0248(5)$ \\
\hline $\mathrm{C} 8$ & 0.7580 & $0.6412(2)$ & $0.88719(14)$ & $0.0378(6)$ \\
\hline H8A & 0.8452 & 0.6152 & 0.9069 & $0.057^{*}$ \\
\hline H8B & 0.6996 & 0.6709 & 0.9252 & $0.057^{*}$ \\
\hline $\mathrm{H} 8 \mathrm{C}$ & 0.7740 & 0.7042 & 0.8528 & $0.057^{*}$ \\
\hline C9 & $0.4734(3)$ & $0.5087(3)$ & $0.89159(16)$ & $0.0525(8)$ \\
\hline H9A & 0.4974 & 0.4249 & 0.8941 & $0.079 *$ \\
\hline H9B & 0.3766 & 0.5168 & 0.8800 & $0.079 *$ \\
\hline $\mathrm{H} 9 \mathrm{C}$ & 0.4914 & 0.5461 & 0.9373 & $0.079 *$ \\
\hline
\end{tabular}
covariance matrix. The cell e.s.d.'s are taken into account individually in the estimation of e.s.d.'s in distances, angles and torsion angles; correlations between e.s.d.'s in cell parameters are only used when they are defined by crystal symmetry. An approximate (isotropic) treatment of cell e.s.d.'s is used for estimating e.s.d.'s involving 1.s. planes.

Fractional atomic coordinates and isotropic or equivalent isotropic displacement parameters $\left(\AA^{2}\right)$

Atomic displacement parameters $\left(\AA^{2}\right)$

\begin{tabular}{lllllll}
\hline & $U^{11}$ & $U^{22}$ & $U^{33}$ & $U^{12}$ & $U^{13}$ & $U^{23}$ \\
\hline Br1 & $0.04164(16)$ & $0.03414(14)$ & $0.03440(17)$ & $0.00799(11)$ & $0.01142(11)$ & $-0.00470(11)$ \\
O1 & $0.0172(9)$ & $0.0434(10)$ & $0.0404(10)$ & $0.0032(7)$ & $-0.0014(7)$ & $-0.0051(8)$ \\
O2 & $0.0191(8)$ & $0.0430(10)$ & $0.0395(10)$ & $0.0057(7)$ & $-0.0011(7)$ & $-0.0108(8)$ \\
N1 & $0.0157(9)$ & $0.0335(11)$ & $0.0285(11)$ & $0.0028(7)$ & $0.0012(8)$ & $-0.0047(9)$ \\
N2 & $0.0195(10)$ & $0.0382(12)$ & $0.0314(12)$ & $0.0014(8)$ & $-0.0023(8)$ & $-0.0082(9)$
\end{tabular}




$\begin{array}{lllllll}\text { C1 } & 0.0194(11) & 0.0238(11) & 0.0241(12) & 0.0008(8) & 0.0040(9) & 0.0012(9) \\ \text { C2 } & 0.0203(12) & 0.0278(11) & 0.0267(13) & 0.0060(9) & 0.0009(9) & 0.0021(10) \\ \text { C3 } & 0.0275(12) & 0.0311(12) & 0.0241(13) & 0.0037(9) & -0.0005(10) & 0.0014(10) \\ \text { C4 } & 0.0282(12) & 0.0256(11) & 0.0255(13) & 0.0023(9) & 0.0092(10) & 0.0001(10) \\ \text { C5 } & 0.0229(12) & 0.0254(11) & 0.0350(14) & 0.0057(9) & 0.0063(10) & 0.0046(10) \\ \text { C6 } & 0.0235(12) & 0.0301(12) & 0.0254(12) & 0.0034(9) & 0.0009(9) & 0.0033(10) \\ \text { C7 } & 0.0223(12) & 0.0273(11) & 0.0249(12) & 0.0011(9) & 0.0003(9) & 0.0032(9) \\ \text { C8 } & 0.0338(14) & 0.0426(15) & 0.0371(15) & -0.0010(11) & -0.0087(12) & -0.0110(12) \\ \text { C9 } & 0.0383(16) & 0.0555(18) & 0.064(2) & -0.0075(13) & 0.0224(15) & -0.0177(16)\end{array}$

Geometric parameters $\left(\AA,{ }^{\circ}\right)$

\begin{tabular}{|c|c|c|c|}
\hline $\mathrm{Br} 1-\mathrm{C} 4$ & $1.904(2)$ & $\mathrm{C} 3-\mathrm{C} 4$ & $1.378(3)$ \\
\hline $\mathrm{O} 1-\mathrm{C} 7$ & $1.228(3)$ & $\mathrm{C} 3-\mathrm{H} 3$ & 0.9500 \\
\hline $\mathrm{O} 2-\mathrm{N} 2$ & $1.410(2)$ & $\mathrm{C} 4-\mathrm{C} 5$ & $1.380(3)$ \\
\hline $\mathrm{O} 2-\mathrm{C} 9$ & $1.421(3)$ & $\mathrm{C} 5-\mathrm{C} 6$ & $1.381(3)$ \\
\hline $\mathrm{N} 1-\mathrm{C} 7$ & $1.361(3)$ & $\mathrm{C} 5-\mathrm{H} 5$ & 0.9500 \\
\hline $\mathrm{N} 1-\mathrm{C} 1$ & $1.411(3)$ & $\mathrm{C} 6-\mathrm{H} 6$ & 0.9500 \\
\hline $\mathrm{N} 1-\mathrm{H} 1 \mathrm{~N}$ & 0.8800 & $\mathrm{C} 8-\mathrm{H} 8 \mathrm{~A}$ & 0.9800 \\
\hline $\mathrm{N} 2-\mathrm{C} 7$ & $1.373(3)$ & $\mathrm{C} 8-\mathrm{H} 8 \mathrm{~B}$ & 0.9800 \\
\hline $\mathrm{N} 2-\mathrm{C} 8$ & $1.448(3)$ & $\mathrm{C} 8-\mathrm{H} 8 \mathrm{C}$ & 0.9800 \\
\hline $\mathrm{C} 1-\mathrm{C} 2$ & $1.388(3)$ & C9-H9A & 0.9800 \\
\hline $\mathrm{C} 1-\mathrm{C} 6$ & $1.391(3)$ & C9-H9B & 0.9800 \\
\hline $\mathrm{C} 2-\mathrm{C} 3$ & $1.380(3)$ & $\mathrm{C} 9-\mathrm{H} 9 \mathrm{C}$ & 0.9800 \\
\hline $\mathrm{C} 2-\mathrm{H} 2$ & 0.9500 & & \\
\hline $\mathrm{N} 2-\mathrm{O} 2-\mathrm{C} 9$ & $108.60(19)$ & $\mathrm{C} 4-\mathrm{C} 5-\mathrm{H} 5$ & 120.5 \\
\hline $\mathrm{C} 7-\mathrm{N} 1-\mathrm{C} 1$ & $123.95(18)$ & C6- $55-\mathrm{H} 5$ & 120.5 \\
\hline $\mathrm{C} 7-\mathrm{N} 1-\mathrm{H} 1 \mathrm{~N}$ & 118.0 & $\mathrm{C} 5-\mathrm{C} 6-\mathrm{C} 1$ & $120.5(2)$ \\
\hline $\mathrm{C} 1-\mathrm{N} 1-\mathrm{H} 1 \mathrm{~N}$ & 118.0 & $\mathrm{C} 5-\mathrm{C} 6-\mathrm{H} 6$ & 119.8 \\
\hline $\mathrm{C} 7-\mathrm{N} 2-\mathrm{O} 2$ & $114.55(17)$ & $\mathrm{C} 1-\mathrm{C} 6-\mathrm{H} 6$ & 119.8 \\
\hline $\mathrm{C} 7-\mathrm{N} 2-\mathrm{C} 8$ & $121.04(19)$ & $\mathrm{O} 1-\mathrm{C} 7-\mathrm{N} 1$ & $125.1(2)$ \\
\hline $\mathrm{O} 2-\mathrm{N} 2-\mathrm{C} 8$ & $112.62(18)$ & $\mathrm{O} 1-\mathrm{C} 7-\mathrm{N} 2$ & $120.1(2)$ \\
\hline $\mathrm{C} 2-\mathrm{C} 1-\mathrm{C} 6$ & $119.4(2)$ & $\mathrm{N} 1-\mathrm{C} 7-\mathrm{N} 2$ & 114.73 (19) \\
\hline $\mathrm{C} 2-\mathrm{C} 1-\mathrm{N} 1$ & 118.07 (19) & $\mathrm{N} 2-\mathrm{C} 8-\mathrm{H} 8 \mathrm{~A}$ & 109.5 \\
\hline $\mathrm{C} 6-\mathrm{C} 1-\mathrm{N} 1$ & $122.5(2)$ & $\mathrm{N} 2-\mathrm{C} 8-\mathrm{H} 8 \mathrm{~B}$ & 109.5 \\
\hline $\mathrm{C} 3-\mathrm{C} 2-\mathrm{C} 1$ & $120.4(2)$ & $\mathrm{H} 8 \mathrm{~A}-\mathrm{C} 8-\mathrm{H} 8 \mathrm{~B}$ & 109.5 \\
\hline $\mathrm{C} 3-\mathrm{C} 2-\mathrm{H} 2$ & 119.8 & $\mathrm{~N} 2-\mathrm{C} 8-\mathrm{H} 8 \mathrm{C}$ & 109.5 \\
\hline $\mathrm{C} 1-\mathrm{C} 2-\mathrm{H} 2$ & 119.8 & $\mathrm{H} 8 \mathrm{~A}-\mathrm{C} 8-\mathrm{H} 8 \mathrm{C}$ & 109.5 \\
\hline $\mathrm{C} 4-\mathrm{C} 3-\mathrm{C} 2$ & $119.2(2)$ & $\mathrm{H} 8 \mathrm{~B}-\mathrm{C} 8-\mathrm{H} 8 \mathrm{C}$ & 109.5 \\
\hline $\mathrm{C} 4-\mathrm{C} 3-\mathrm{H} 3$ & 120.4 & $\mathrm{O} 2-\mathrm{C} 9-\mathrm{H} 9 \mathrm{~A}$ & 109.5 \\
\hline $\mathrm{C} 2-\mathrm{C} 3-\mathrm{H} 3$ & 120.4 & $\mathrm{O} 2-\mathrm{C} 9-\mathrm{H} 9 \mathrm{~B}$ & 109.5 \\
\hline $\mathrm{C} 3-\mathrm{C} 4-\mathrm{C} 5$ & $121.5(2)$ & $\mathrm{H} 9 \mathrm{~A}-\mathrm{C} 9-\mathrm{H} 9 \mathrm{~B}$ & 109.5 \\
\hline $\mathrm{C} 3-\mathrm{C} 4-\mathrm{Br} 1$ & $118.85(18)$ & $\mathrm{O} 2-\mathrm{C} 9-\mathrm{H} 9 \mathrm{C}$ & 109.5 \\
\hline $\mathrm{C} 5-\mathrm{C} 4-\mathrm{Br} 1$ & $119.62(16)$ & $\mathrm{H} 9 \mathrm{~A}-\mathrm{C} 9-\mathrm{H} 9 \mathrm{C}$ & 109.5 \\
\hline $\mathrm{C} 4-\mathrm{C} 5-\mathrm{C} 6$ & $119.0(2)$ & $\mathrm{H} 9 \mathrm{~B}-\mathrm{C} 9-\mathrm{H} 9 \mathrm{C}$ & 109.5 \\
\hline $\mathrm{C} 9-\mathrm{O} 2-\mathrm{N} 2-\mathrm{C} 7$ & $116.7(2)$ & $\mathrm{Br} 1-\mathrm{C} 4-\mathrm{C} 5-\mathrm{C} 6$ & $178.85(16)$ \\
\hline
\end{tabular}




$\begin{array}{llll}\mathrm{C} 9-\mathrm{O} 2-\mathrm{N} 2-\mathrm{C} 8 & -99.8(2) & \mathrm{C} 4-\mathrm{C} 5-\mathrm{C} 6-\mathrm{C} 1 & 0.0(3) \\ \mathrm{C} 7-\mathrm{N} 1-\mathrm{C} 1-\mathrm{C} 2 & -141.5(2) & \mathrm{C} 2-\mathrm{C} 1-\mathrm{C} 6-\mathrm{C} 5 & 2.3(3) \\ \mathrm{C} 7-\mathrm{N} 1-\mathrm{C} 1-\mathrm{C} 6 & 40.2(3) & \mathrm{N} 1-\mathrm{C} 1-\mathrm{C} 6-\mathrm{C} 5 & -179.4(2) \\ \mathrm{C} 6-\mathrm{C} 1-\mathrm{C} 2-\mathrm{C} 3 & -2.8(3) & \mathrm{C} 1-\mathrm{N} 1-\mathrm{C} 7-\mathrm{O} 1 & -1.2(4) \\ \mathrm{N} 1-\mathrm{C} 1-\mathrm{C} 2-\mathrm{C} 3 & 178.9(2) & \mathrm{C} 1-\mathrm{N} 1-\mathrm{C} 7-\mathrm{N} 2 & -177.6(2) \\ \mathrm{C} 1-\mathrm{C} 2-\mathrm{C} 3-\mathrm{C} 4 & 0.9(3) & \mathrm{O} 2-\mathrm{N} 2-\mathrm{C} 7-\mathrm{O} 1 & 165.9(2) \\ \mathrm{C} 2-\mathrm{C} 3-\mathrm{C} 4-\mathrm{C} 5 & 1.4(3) & \mathrm{C} 8-\mathrm{N} 2-\mathrm{C} 7-\mathrm{O} 1 & 25.7(3) \\ \mathrm{C} 2-\mathrm{C} 3-\mathrm{C} 4-\mathrm{Br} 1 & -179.30(17) & \mathrm{O} 2-\mathrm{N} 2-\mathrm{C} 7-\mathrm{N} 1 & -17.6(3) \\ \mathrm{C} 3-\mathrm{C} 4-\mathrm{C} 5-\mathrm{C} 6 & -1.9(3) & \mathrm{C} 8-\mathrm{N} 2-\mathrm{C} 7-\mathrm{N} 1 & -157.8(2)\end{array}$

Hydrogen-bond geometry $\left(A,{ }^{\circ}\right)$

$\mathrm{Cg} 1$ is the centroid of the $\mathrm{C} 1-\mathrm{C} 6$ ring.

\begin{tabular}{lllll}
\hline$D-\mathrm{H} \cdots A$ & $D-\mathrm{H}$ & $\mathrm{H} \cdots A$ & $D \cdots A$ & $D-\mathrm{H} \cdots A$ \\
\hline $\mathrm{N} 1-\mathrm{H} 1 N \cdots \mathrm{O} 1^{\mathrm{i}}$ & 0.88 & 2.39 & $3.130(2)$ & 142 \\
$\mathrm{C} 2-\mathrm{H} 2 \cdots \mathrm{O} 1^{\mathrm{i}}$ & 0.95 & 2.42 & $3.217(3)$ & 142 \\
$\mathrm{C} 9-\mathrm{H} 9 A \cdots C g 1^{\mathrm{i}}$ & 0.98 & 2.99 & $3.477(3)$ & 112 \\
\hline
\end{tabular}

Symmetry code: (i) $x-1 / 2, y,-z+3 / 2$. 